\title{
Evaluation of inter-departmental variability of ejection fraction and cardiac volumes in myocardial perfusion scintigraphy using simulated data
}

Elin Trägårdh ${ }^{1 *}$, Michael Ljungberg ${ }^{2}$, Lars Edenbrandt ${ }^{1}$, Eva Örndahl ${ }^{3}$, Lena Johansson ${ }^{4}$, Agneta Gustafsson ${ }^{5}$, Cathrine Jonsson ${ }^{5}$, Jessica Hagerman ${ }^{1}$, Katrine Riklund ${ }^{6}$ and David Minarik ${ }^{7}$

\section{* Correspondence:}

elin.tragardh@med.lu.se

${ }^{1}$ Clinical Physiology and Nuclear Medicine, Skåne University Hospital, Lund University Hospital, Inga Marie Nilssons gata 49, 20502 Malmö, Sweden

Full list of author information is available at the end of the article

\begin{abstract}
Background: Myocardial perfusion scintigraphy (MPS) is a clinically useful noninvasive imaging modality for diagnosing patients with suspected coronary artery disease. By utilizing gated MPS, the end diastolic volume (EDV) and end systolic volume (ESV) can be measured and the ejection fraction (EF) calculated, which gives incremental prognostic value compared with assessment of perfusion only. The aim of this study was to evaluate the inter-departmental variability of EF, ESV, and EDV during gated MPS in Sweden.

Methods: Seventeen departments were included in the study. The SIMIND Monte Carlo (MC) program together with the XCAT phantom was used to simulate three patient cases with different EDV, ESV, and EF. Individual simulations were performed for each department, corresponding to their specific method of performing MPS. Images were then sent to each department and were evaluated according to clinical routine. EDV, ESV, and EF were reported back.

Results: There was a large underestimation of EDV and ESV for all three cases. Mean underestimation for EDV varied between 26\% and 52\% and for ESV between 15\% and $60 \%$. EF was more accurately measured, but mean bias still varied between an underestimation of $24 \%$ to an overestimation of $14 \%$. In general, the intra-departmental variability for EDV, ESV, and EF was small, whereas inter-departmental variability was larger.
\end{abstract}

Conclusions: Left ventricular volumes were generally underestimated, whereas EF was more accurately estimated. There was, however, large inter-departmental variability.

Keywords: External quality assessment; Monte Carlo simulations; SPECT; Myocardial perfusion imaging

\section{Background}

Myocardial perfusion scintigraphy (MPS) is widely regarded as a clinically useful noninvasive imaging modality for diagnosing patients with suspected coronary artery disease. The diagnostic accuracy is high, and risk stratification has been well validated [1-3]. By utilizing gated MPS, the end diastolic volume (EDV) and end systolic volume 
(ESV) can be measured and the ejection fraction (EF) can thus be calculated. Assessment of left ventricular volumes and function are important parameters in patients with coronary artery disease. The information from gated MPS has been shown to have incremental prognostic value compared with assessment of perfusion only $[4,5]$. Decreased EF and increased left ventricular volumes without any perfusion defects are seen in, for example, some cases of cardiomyopathy. A stress perfusion defect with decreased function compared with the resting images is indicative of postischemic myocardial stunning. Lower EF and larger volumes after stress compared with rest are signs of severe ischemia.

It is vital that different departments measure the same EF and left ventricular volumes. The aim of this study was to evaluate the inter-departmental variability of EF, ESV, and EDV obtained from gated MPS in Sweden. The study was performed as a part of a national QA program in nuclear medicine, initiated and managed by Equalis $A B$ [6], a non-profit company providing external quality assessment of laboratory investigations within the Swedish healthcare.

Evaluation of inter-departmental variability of EF, ESV, and EDV has been performed by Verberne et al. [7]. They used a physical phantom [8], which had a dynamic heart and modifiable ESV and EDV. This method of evaluation requires that each participating department owns its own phantom or that one or more phantoms are sent around. Each department also has to perform several measurements including phantom preparation that requires both time and effort. To overcome the inconvenience of constructing and sending a physical phantom around, we have instead created Monte Carlo simulated gated MPS studies for distribution. By using the Monte Carlo method, we are able to use a more realistic digital phantom with more degrees of freedom regarding the shape of the phantom, physiological movements due to breathing, and the motion of the heart.

\section{Methods}

Twenty-six departments were enrolled to participate in the evaluation. Four were not able to participate because they either use the new CZT cameras or use special heart collimators which at the moment are not supported by the Monte Carlo program. Five departments did not respond/had troubles reading the data files. Seventeen departments ended up participating in the evaluation.

Each participating department reported the method they use to perform MPS, e.g. the camera system, camera settings (energy window, matrix size, number of projection angles, etc.), and the administered radioactivity. All reported parameters are listed in Table 1. The SIMIND Monte Carlo program [9] together with the XCAT anthropomorphic computer phantom [10] was then used to simulate projection data. Simulations were performed for each department using their characteristic camera settings. Three different patient cases were simulated as described in Table 2. The cases were chosen to cover as much of the patient spectrum that is normally encountered in the clinic with the three cases. For each patient case, 32 instances of the XCAT phantom were created with the heart in different positions in the cardiac cycle and different spatial positions in the thoracic cavity due to breathing. For each department, all the 32 phantoms were simulated and the results were summed corresponding to each 
Table 1 Parameters that were provided from the different departments for the simulations

\begin{tabular}{lc}
\hline Camera system & Starting angle \\
\hline Collimator & Total rotation \\
Crystal thickness & Number of projections \\
Energy window & Time per projection \\
Matrix size & Number of time frames \\
Pixel size & Administered activity \\
\hline
\end{tabular}

department's method of gating, i.e. if 8 or 16 gates were used. The biokinetics was taken from [11], and the fraction of the administered activity in the heart after $1 \mathrm{~h}$ was set to $1.2 \%$. Here, we did not take into account eventual differences between tetrofosmin and sestamibi. In order to mimic real measurements, the simulations were performed with sufficient histories to generate noiseless data. Poisson noise was then added after the simulations, corresponding to a count level representative for the administered activity and acquisition time for each department and the aforementioned biokinetics. The final projections were converted to DICOM format and sent to the departments, which evaluated the simulations as according to their methods of tomographic reconstruction and ESV, EDV, and EF quantifications. The departments used different software packages for the evaluation, according to clinical routine (reported use of software packages for different departments: Quantitative Gated SPECT (Cedars Sinai Los Angeles, CA, USA; QPS), AutoQuant (Philips, Andover, MA, USA), EXINI Heart (EXINI Diagnostics, Lund, Sweden), Emory Cardiac Toolbox (Emory University Medical Center, Atlanta, GA, USA)). The evaluation was restricted to non-attenuation corrected images and reconstruction without collimator detector response compensations because of difficulties of generating DICOM images with the required header information for each gamma camera vendor. The methods used by the departments are listed in Table 3; however, some departments did not report their methods. Five departments contributed with one response each and 12 departments contributed with multiple responses since more than one technologist performed the evaluation.

\section{Statistical analysis}

Values of reported EDV, ESV, and EF are given as mean and 95\% confidence interval (CI). Mean bias as well as mean absolute bias was calculated. One sample $t$-test was used to determine differences between reported values and the true simulated value for EDV, ESV, and EF. When more than one value was reported from one department, the

Table 2 Patient characteristics for the three cases

\begin{tabular}{lccc}
\hline & Case $\mathbf{1}$ & Case 2 & Case 3 \\
\hline Sex & Female & Male & Female \\
Length $(\mathrm{cm})$ & 160 & 182 & 171 \\
Weight $(\mathrm{kg})$ & 55 & 102 & 68 \\
EF $(\%)$ & 53 & 37 & 62 \\
EDV $(\mathrm{mL})$ & 50 & 230 & 91 \\
ESV $(\mathrm{mL})$ & 24 & 143 & 35 \\
\hline
\end{tabular}




\begin{tabular}{|c|c|c|c|c|c|}
\hline Department number & $\begin{array}{l}\text { Reconstruction } \\
\text { algorithm }\end{array}$ & $\begin{array}{l}\text { Iteration } \\
\text { updates }\end{array}$ & Filter & Cut-off frequency/width ${ }^{a}$ & $\begin{array}{c}\text { Evaluation } \\
\text { software }\end{array}$ \\
\hline 1 & N/A & & & & \\
\hline 2 & FBP & & Butterworth & 0.52 & ECToolbox \\
\hline 3 & OSEM & 80 & Butterworth & 0.45 & QGS \\
\hline 4 & OSEM & N/A & Hanning & & QGS \\
\hline 5 & OSEM & 48 & Butterworth & 0.4 & AutoQuant \\
\hline 6 & N/A & & & & \\
\hline 7 & FBP & & Butterworth & 0.35 & QGS \\
\hline 8 & N/A & & & & \\
\hline 9 & FBP & & Butterworth & 0.35 & Exini heart \\
\hline 10 & FBP & & Butterworth & 0.65 & QGS \\
\hline 11 & N/A & & & & \\
\hline 12 & FBP & & Butterworth & 0.4 & QGS \\
\hline 13 & FBP & & Butterworth & 0.9 & QGS \\
\hline 14 & FBP & & Butterworth & 0.52 & QGS \\
\hline 15 & OSEM & 120 & Butterworth & 0.4 & QGS \\
\hline 16 & FBP & & Butterworth & 0.4 & ECtoolbox \\
\hline 17 & OSEM & 32 & 3D Gaussian & 0.8 & QGS \\
\hline
\end{tabular}

${ }^{\mathrm{a}}$ Cut-off frequency for Butterworth filter is in unit of Nyquist frequency and SD for 3D Gaussian filter is in pixels. FBP filtered back projection, OSEM ordered subset expectation maximization.

mean value from that department was used in the $t$-test. Statistical significance was set at 0.05. Statistical analysis was carried out using MedCalc for Windows, version 12.7.7.0 (MedCalc Software, Ostend, Belgium).

\section{Results}

The results are displayed in Figure 1 and Table 4. There was a large underestimation of both EDV and ESV for all three cases. The largest underestimation was seen for case 1 (female with a small heart) and the smallest underestimation for case 2 (male with a large heart). Mean underestimation for EDV varied between $26 \%$ and $52 \%$ and for ESV between $15 \%$ and $60 \%$. EF was more accurately estimated, but mean bias still varied between an underestimation of $24 \%$ to an overestimation of $14 \%$. The mean absolute bias was $17 \%, 24 \%$, and $9 \%$ for cases 1 to 3, respectively. In general, the intra-departmental variability for EDV, ESV, and EF was small, whereas inter-departmental variability was larger (Figure 1). The outlier in department no. 1 could be due to a typo.

\section{Discussion}

Our study shows that there is a large inter-departmental variability of EF, EDV, and ESV estimations in nuclear medicine departments in Sweden, but the intradepartmental variability was found to be small. We found a large underestimation of EDV and ESV, but since the underestimation of EDV and ESV was of similar order, the EF estimates were more accurate, although not satisfactory. The mean bias varied from $14 \%$ for the small heart, where 14 out of 17 departments overestimated the EF, to $-24 \%$ 


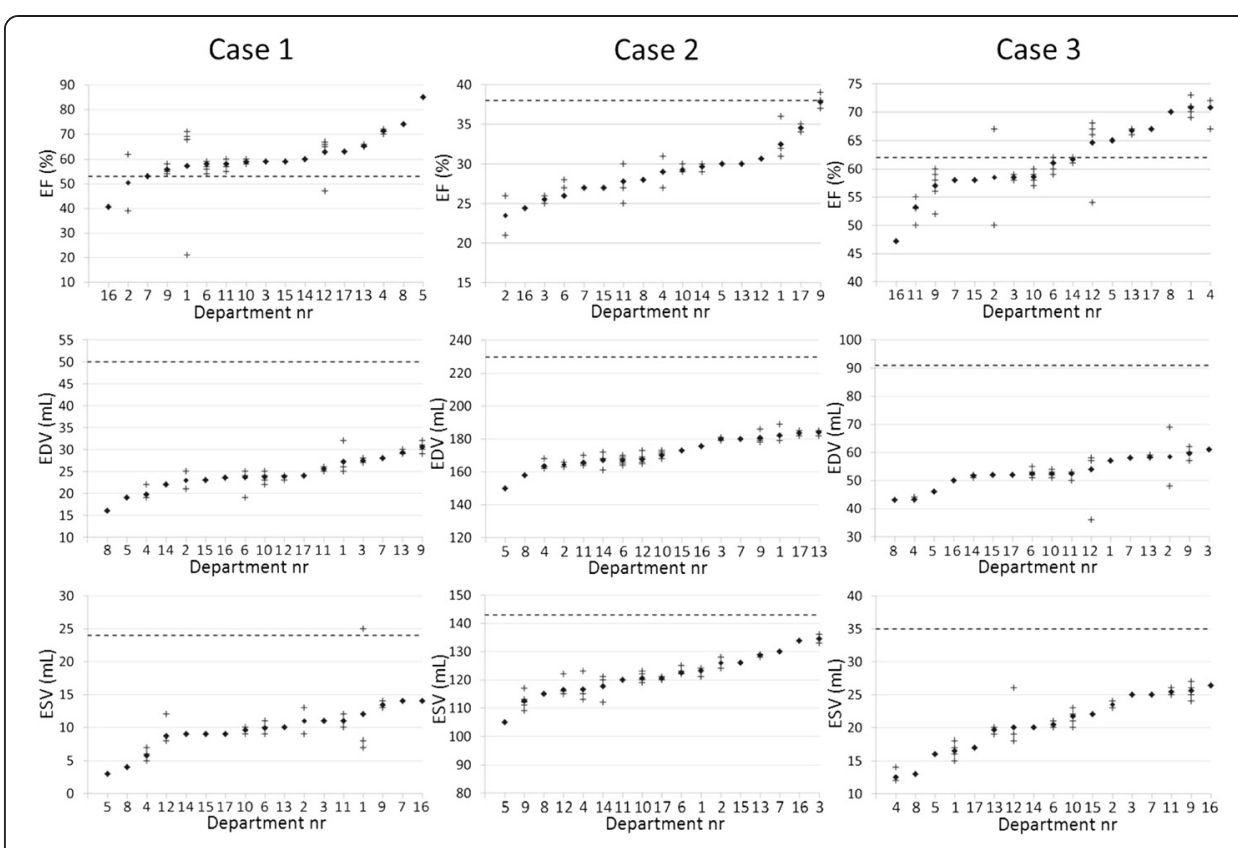

Figure 1 Dotted lines represent true values. Plus signs represent answers from individual technologists (provided by 12 departments) and diamond signs represent the department mean.

for the large heart, where all but one underestimated the EF. For the normal heart, the mean bias was around $1 \%$ but the mean absolute bias was however $9 \%$, indicating that the accuracy of the EF estimates also for the normal sized heart was low. Since the evaluation of EF to a large extent is automated, there should be a small intradepartmental variability and we are recommending that departments that have a larger intra-departmental variability should perform an internal quality control of their method. The large inter-departmental variability is however of concern, both for cardiac volumes and for EF. The volume estimates of the LV depend on the spatial resolution of the final images and the algorithm used to outline the LV. The spatial resolution depends on several parameters such as choice of collimator, pixel size, noise level, which determines eventual pre- or post-reconstruction filters, and the reconstruction algorithm. The noise level in turn is dependent on administered activity, measurement time per frame, number of frames per heart cycle, number of projections, and pixel size. Different software packages use different algorithms to outline the LV, and these are more or less prone to give different results depending on the resolution in the input image. It was beyond the scope of this study to evaluate to what extent each parameter affects the final result because the statistical basis was too small. However, some

Table 4 Mean values, $95 \% \mathrm{Cl}$, mean bias, and $p$ values for all departments

\begin{tabular}{lcccccccccc}
\hline & \multicolumn{3}{c}{ Case 1 } & \multicolumn{3}{c}{ Case 2 } & \multicolumn{3}{c}{ Case 3 } \\
\hline & $E F$ & $E D V$ & $E S V$ & $E F$ & $E D V$ & $E S V$ & $E F$ & $E D V$ & $E S V$ \\
$\begin{array}{l}\text { Mean all departments } \\
(\mathrm{mL})\end{array}$ & 61 & 24 & 10 & 29 & 171 & 122 & 62 & 53 & 21 \\
$95 \% \mathrm{Cl}(\mathrm{mL})$ & 42 to 79 & 17 to 31 & 4 to 16 & 22 to 36 & 153 to 190 & 107 to 136 & 49 to 74 & 43 to 63 & 12 to 29 \\
Mean bias (\%) & 14 & -52 & -60 & -24 & -26 & -15 & -1 & -42 & -41 \\
$p$ value & 0.005 & $<0.001$ & $<0.001$ & $<0.001$ & $<0.001$ & $<0.001$ & 0.77 & $<0.001$ & $<0.001$ \\
\hline
\end{tabular}


tendencies can be extracted from the data. It can be seen that ECToolbox generally gave lower EF than other softwares. Also, departments using OSEM or FBP with higher cut-off frequency gave more accurate volume estimates of the small heart due to better resolution. Regarding the cases with normal and large heart, the results were more arbitrary, indicating that it is a combination of measurement protocol, reconstruction parameters, and evaluation software that determine the outcome of the volume estimates.

In the study by Verberne et al. [7], they used a physical phantom that included a dynamic heart model able to define different ESV, EDV, and heart wall thickness but the phantom could not mimic physical properties such as heart motion due to breathing. This phantom was distributed to 35 nuclear medicine departments for individual measurements using three different settings; with an ESV of 50, 90, and $120 \mathrm{~mL}$ respectively and an EDV of 120, 160, and $190 \mathrm{~mL}$ respectively, which give an EF of 58\%, 44\%, and 37\%, respectively. Results showed that the EDV and ESV were on average underestimated by 1 to $65 \mathrm{~mL}$ respectively and the EF was overestimated by $1 \%$ to $15 \%$, while our results showed both under- and overestimation of the EF. This difference was probably due the fact that we had a more differentiated phantom population. Also, in their study, only the QGS software package was used to quantify the measurements; whereas in our study, the contributing departments used their clinical routine software packages.

Several previous studies have compared LV volumes as quantified by different algorithms to cardiac magnetic resonance imaging, regarded as the reference standard. In the majority of the studies, EDV and ESV by MPS were underestimated [12-18]. EDV by MPS has also been shown to range from overestimation to underestimation compared to magnetic resonance imaging depending on the software package used [19-21]. One study has shown an overestimation in both EDV and ESV by MPS [22]. An explanation for the differences found in these studies, apart from differences in the algorithms for calculating MPS volumes, could be different magnetic resonance sequences. It has been shown that older turbo gradient echo imaging shows significantly smaller EDV and ESV compared to current standard steady-state free precession imaging [23]. The results obtained from the studies using newer magnetic resonance imaging technique are in accordance with our results.

Based on the results of our and others studies, it should be debated whether ESV and EDV should be provided in a clinical final report of the MPS. The American Society of Nuclear Cardiology conclude in their consensus statement [24] on reporting of myocardial perfusion imaging studies that a quantitative value of left ventricular EF should be included within the report. For EF greater than $60 \%$, the actual calculated number should be included in the report and mention made of overestimation in patients with small hearts. Ventricular volumes may be reported (optional). The European Association of Nuclear Medicine and European Society of Cardiology state in their procedural guidelines for myocardial perfusion imaging [25] that EF and volumes should be reported together and that caution should be exercised in reporting apparently spurious values of these parameters. It is also stated that reporting of volumes may preferably be indexed according to body surface area, since the reference values have a narrower range. We believe that if physicians want to state estimated EF and volumes in their reports, they should be careful to use normal limits that have been established for the same MPS technique and software tool as used at their department. 
This study has some limitations. One is that we only enrolled departments that use standard gamma cameras with parallel hole collimators. Newer technologies, such as the new semiconductor cameras (CZT cameras) and special collimators, such as the IQ-SPECT system (Siemens, Erlangen, Germany) could not be simulated in the present version of the SIMIND code. Also, only non-attenuation corrected images were used, since departmental specific attenuation maps generated from CT were difficult to create. Thus, the results from the study do not exactly represent the clinical situation in Sweden but indicate tendencies of the large variability. The SIMIND Monte Carlo program is a useful tool to test an imaging method when the aim is to test the robustness of the method in a patient-like situation. However, a simulation is not entirely equal to a real measurement since the effects of e.g. non-perfect intrinsic spatial uniformity and linearity is not modeled. Also, eventual patient movement in addition to breathing and beating heart is not modeled. The heart in the phantoms used in the study is derived from one real patient and scaled to different sizes. Although attenuation conditions such as amount of fat, size of the heart, and breasts differ, the shape of the heart is the same in all cases.

\section{Conclusion}

In our study, we have used the MC method for a multi-center quality control study of MPI, both in terms of differences between departments and the general accuracy of the method. We have confirmed the results from previous studies that left ventricular volumes are underestimated in MPS, whereas EF is more accurately estimated. There was, however, large inter-departmental variability, which needs to be further addressed.

Competing interests

EÖ is employed by Equalis AB. LE is a shareholder and is employed by EXINI Diagnostics AB.

\section{Authors' contributions}

All authors participated in the design of the study. ML and DM performed the MC simulations. EÖ coordinated the project. ET and DM drafted the manuscript. All authors read and approved the final manuscript.

\footnotetext{
Author details

${ }^{1}$ Clinical Physiology and Nuclear Medicine, Skåne University Hospital, Lund University Hospital, Inga Marie Nilssons gata 49, 20502 Malmö, Sweden. ²Department of Medical Radiation Physics, Lund University, Lund, Sweden. ${ }^{3}$ Equalis AB, Uppsala, Sweden. ${ }^{4}$ Clinical Physiology, Central Hospital, Karlstad, Sweden. ${ }^{5}$ Department of Medical Physics, Karolinska University Hospital, Stockholm, Sweden. 'Department of Radiation Sciences, Umeå University, Umeå, Sweden.

${ }^{7}$ Radiation Physics, Skåne University Hospital, Lund University, Malmö, Sweden.
}

Received: 15 September 2014 Accepted: 9 December 2014

Published online: 23 January 2015

References

1. Shaw $\amalg$, Hage FG, Berman DS, Hachamovitch R, Iskandrian A. Prognosis in the era of comparative effectiveness research: where is nuclear cardiology now and where should it be? J Nucl Cardiol. 2012;19:1026-43.

2. Hachamovitch R, Berman DS, Shaw L, Kiat H, Cohen I, Cabico JA, Friedman J, Diamond GA. Incremental prognostic value of myocardial perfusion single photon emission computed tomography for the prediction of cardiac death: differential stratification for risk of cardiac death and myocardial infarction. Circulation. 1998;97:535-43.

3. Hachamovitch R, Berman DS, Kiat H, Cohen I, Cabico JA, Friedman J, Diamond GA. Exercise myocardial perfusion SPECT in patients without known coronary artery disease: incremental prognostic value and use in risk stratification. Circulation. 1996:93:905-14.

4. Lima RS, Watson DD, Goode AR, Siadaty MS, Ragosta M, Beller GA, Samady H. Incremental value of combined perfusion and function over perfusion alone by gated SPECT myocardial perfusion imaging for detection of severe three-vessel coronary artery disease. J Am Coll Cardiol. 2003;42:64-70.

5. Sharir T, Germano G, Kavanagh PB, Lai S, Cohen I, Lewin HC, Friedman JD, Zellweger MJ, Berman DS. Incremental prognostic value of post-stress left ventricular ejection fraction and volume by gated myocardial perfusion single photon emission computed tomography. Circulation. 1999:100:1035-42.

6. Equalis $\mathrm{AB}[\mathrm{http}: / / w w w . e q u a l i s . s e / e n / s t a r t . a s p x]$ 
7. Verberne HJ, Dibbets-Schneider P, Spijkerboer A, Stokkel M, van Eck-Smit BL, Sokole EB. Multicenter intercomparison assessment of consistency of left ventricular function from a gated cardiac SPECT phantom. J Nucl Cardiol. 2006;13:801-10.

8. Visser JJ, Sokole EB, Verberne HJ, Habraken JB, van de Stadt HJ, Jaspers JE, Shehata M, Heeman PM, van Eck-Smit BL. A realistic 3-D gated cardiac phantom for quality control of gated myocardial perfusion SPET: the Amsterdam gated (AGATE) cardiac phantom. Eur J Nucl Med Mol Imaging. 2004;31:222-8.

9. Ljungberg M, Strand SE. A Monte Carlo program for the simulation of scintillation camera characteristics. Comput Methods Programs Biomed. 1989;29:257-72.

10. Segars WP, Sturgeon G, Mendonca S, Grimes J, Tsui BM. 4D XCAT phantom for multimodality imaging research. Med Phys. 2010;37:4902-15.

11. Higley B, Smith FW, Smith T, Gemmell HG, Das Gupta P, Gvozdanovic DV, Graham D, Hinge D, Davidson J, Lahiri A. Technetium-99 m-1,2-bis[bis(2-ethoxyethyl) phosphino]ethane: human biodistribution, dosimetry and safety of a new myocardial perfusion imaging agent. J Nucl Med. 1993;34:30-8.

12. Soneson H, Hedeer F, Arevalo C, Carlsson M, Engblom H, Ubachs JF, Arheden H, Heiberg E. Development and validation of a new automatic algorithm for quantification of left ventricular volumes and function in gated myocardial perfusion SPECT using cardiac magnetic resonance as reference standard. J Nucl Cardiol. 2011;18:874-85.

13. Mistry N, Halvorsen S, Hoffmann P, Muller C, Bohmer E, Kieldsen SE, Bjornerheim R. Assessment of left ventricular function with magnetic resonance imaging vs. echocardiography, contrast echocardiography, and single-photon emission computed tomography in patients with recent ST-elevation myocardial infarction. Eur J Echocardiogr. 2010;11:793-800

14. Hedeer F, Palmer J, Arheden H, Ugander M. Gated myocardial perfusion SPECT underestimates left ventricular volumes and shows high variability compared to cardiac magnetic resonance imaging - a comparison of four different commercial automated software packages. BMC Med Imaging. 2010;10:10.

15. Chan J, Jenkins C, Khafagi F, Du L, Marwick TH. What is the optimal clinical technique for measurement of left ventricular volume after myocardial infarction? A comparative study of 3-dimensional echocardiography, single photon emission computed tomography, and cardiac magnetic resonance imaging. J Am Soc Echocardiogr. 2006;19:192-201.

16. Persson $\mathrm{E}$, Carlsson $\mathrm{M}$, Palmer J, Pahlm O, Arheden $\mathrm{H}$. Evaluation of left ventricular volumes and ejection fraction by automated gated myocardial SPECT versus cardiovascular magnetic resonance. Clin Physiol Funct Imaging. 2005;25:135-41.

17. Bax JJ, Lamb H, Dibbets P, Pelikan H, Boersma E, Viergever EP, Germano G, Vliegen HW, de Roos A, Pauwels EK, Van der Wall EE. Comparison of gated single-photon emission computed tomography with magnetic resonance imaging for evaluation of left ventricular function in ischemic cardiomyopathy. Am J Cardiol. 2000;86:1299-305.

18. Bavelaar-Croon CD, Kayser HW, van der Wall EE, de Roos A, Dibbets-Schneider P, Pauwels EK, Germano G, Atsma DE. Left ventricular function: correlation of quantitative gated SPECT and MR imaging over a wide range of values. Radiology. 2000;217:572-5.

19. Winz OH, Meyer PT, Knollmann D, Lipke CS, Kuhl HP, Oelve C, Schaefer WM. Quantification of left ventricular volumes and ejection fraction from gated 99mTc-MIBI SPECT: MRI validation of the EXINI heart software package. Clin Physiol Funct Imaging. 2009;29:89-94.

20. Stegger L, Lipke CS, Kies P, Nowak B, Schober O, Buell U, Schafers M, Schaefer WM. Quantification of left ventricular volumes and ejection fraction from gated 99mTC-MIBI SPECT: validation of an elastic surface model approach in comparison to cardiac magnetic resonance imaging, 4D-MSPECT and QGS. Eur J Nucl Med Mol Imaging. 2007;34:900-9.

21. Lipke CS, Kuhl HP, Nowak B, Kaiser HJ, Reinartz P, Koch KC, Buell U, Schaefer WM. Validation of 4D-MSPECT and QGS for quantification of left ventricular volumes and ejection fraction from gated 99mTc-MIBI SPET: comparison with cardiac magnetic resonance imaging. Eur J Nucl Med Mol Imaging. 2004;31:482-90.

22. Faber TL, Vansant JP, Pettigrew Rl, Galt JR, Blais M, Chatzimavroudis G, Cooke CD, Folks RD, Waldrop SM, Gurtler-Krawczynska E, Wittry MD, Garcia EV. Evaluation of left ventricular endocardial volumes and ejection fractions computed from gated perfusion SPECT with magnetic resonance imaging: comparison of two methods. J Nucl Cardiol. 2001;8:645-51.

23. Alfakih K, Plein S, Thiele H, Jones T, Ridgway JP, Sivananthan MU. Normal human left and right ventricular dimensions for MRI as assessed by turbo gradient echo and steady-state free precession imaging sequences. J Magn Reson Imaging. 2003;17:323-9.

24. Hendel RC, Wackers FJ, Berman DS, Ficaro E, DePuey EG, Klein L, Cerqueira M. American Society of Nuclear Cardiology consensus statement: reporting of radionuclide myocardial perfusion imaging studies. J Nucl Cardiol. 2006;13:e152-156.

25. Hesse B, Tagil K, Cuocolo A, Anagnostopoulos C, Bardies M, Bax J, Bengel F, Busemann Sokole E, Davies G, Dondi M, Edenbrandt L, Franken P, Kjaer A, Knuuti J, Lassmann M, Ljungberg M, Marcassa C, Marie PY, McKiddie F, O'Connor M, Prvulovich E, Underwood R, van Eck-Smit B. EANM/ESC procedural guidelines for myocardial perfusion imaging in nuclear cardiology. Eur J Nucl Med Mol Imaging. 2005;32:855-97. 